\title{
Dynamical Properties of Spin-Orbital Chains in a Magnetic Field
}

\author{
Weiqiang Yu and Stephan Haas \\ Department of Physics and Astronomy, University of Southern California, Los Angeles, CA \\ 90089-0484
}

(October 28, 2018)

\begin{abstract}
The excitation spectrum of the one-dimensional spin-orbital model in a magnetic field is studied, using a recently developed dynamical density matrix renormalization group technique. The method is employed on chains with up to 80 sites, and examined for test cases such as the spin- $1 / 2$ antiferromagnetic Heisenberg chain, where the excitation spectrum is known exactly from the Bethe Ansatz. In the spin-orbital chain, the characteristic dynamical response depends strongly on the model parameters and the applied magnetic field. The coupling between the spin and orbital degrees of freedom is found to influence the incommensuration at finite magnetizations. In the regions of the phase diagram with only massive spin and orbital excitations, a finite field is required to overcome the spin gap. An incommensurate orbital mode is found to become massless in this partially spin-polarized regime, indicating a strong coupling between the two degrees of freedom. In the critical region with three elementary gapless excitations, a prominent particle-hole excitation is observed at higher energies, promoted by the biquadratic term in the model Hamiltonian of the spin-orbital chain.
\end{abstract}




\section{INTRODUCTION}

There is a wide variety of quasi-one-dimensional spin systems with short-ranged and quasi-long-ranged quantum liquid states. Examples for short-ranged compounds include the spin-Peierls chain, even-leg spin-1/2 Heisenberg ladders, and integer-spin Heisenberg chains. In contrast, half-odd-integer-spin chains and odd-leg spin-1/2 Heisenberg ladders are known to have a quantum critical ground state with quasi-long-range-ordered spin correlations. Many quasi-one-dimensional models, such as the antiferromagnetic spin-1/2 Heisenberg chain with frustrating longer-range exchange interactions, have a phase diagram which contains both types of disordered ground state, depending on the parameters. Particular recent attention has focussed on spin-1/2 Heisenberg chains coupled to orbital degrees of freedom. [1-9] These systems are known to have a rich phase diagram, containing shortranged and quasi-long-ranged regions, including an $\mathrm{SU}(4)$-symmetric integrable point at a particular combination of the exchange parameters.

In this work, we study the spin and orbital excitation spectra of spin-orbital chains in a magnetic field. By applying a newly developed dynamical density matrix renormalization group (DMRG) technique, [10,11] it is found that the admixture of the orbital degrees of freedom has a profound influence on both the field-dependent incommensuration, and the dispersion and spectral weight of the spin triplet modes, although only the spins couple directly to the applied magnetic field. Furthermore, this study is intended to provide useful information for inelastic neutron scattering studies on orbitally degenerate materials such as $\mathrm{Na}_{2} \mathrm{Ti}_{2} \mathrm{Sb}_{2} \mathrm{O}$ [12], where the d-electron of the $\mathrm{Ti}^{3+}$ ion occupies an $e_{g}$ doublet, and $\mathrm{NaV}_{2} \mathrm{O}_{5}$, where orbital degeneracy appears to arise from the coupling of two types of $\mathrm{VO}_{5}$ chains. [1.13 16] As long as experimentally available magnetic fields ( 30 Tesla) are sufficiently large to overcome the respective spin gaps, our results for the dynamical properties of the incommensurate phases in the one-dimensional spin-orbital model may be tested on these or suitably related compounds.

In this paper, the Hamiltonian of the one-dimensional spin-orbital model in a magnetic 
field is studied. This can be written as

$$
H=J_{1} \sum_{i=1}^{N} \mathbf{S}_{i} \cdot \mathbf{S}_{i+1}+J_{2} \sum_{i=1}^{N} \mathbf{T}_{i} \cdot \mathbf{T}_{i+1}+K \sum_{i=1}^{N}\left(\mathbf{S}_{i} \cdot \mathbf{S}_{i+1}\right)\left(\mathbf{T}_{i} \cdot \mathbf{T}_{i+1}\right)-h \sum_{i=1}^{N} S_{i}^{z},
$$

where the spin-1/2 operators $\mathbf{S}_{i}$ and the pseudo-spin- $1 / 2$ operators $\mathbf{T}_{i}$ describe the spins and orbits at a site $i$. [17] The first two terms are the Hamiltonians for (pseudo-)spin-1/2 Heisenberg chains with exchange constants $J_{1}$ for the spins and $J_{2}$ for the orbitals. The third term couples these two sets of degrees of freedom with a constant $K$. The fourth term is the Zeeman coupling of the spins to an external magnetic field aligned with the $z$-direction. In the absence of a magnetic field, this Hamiltonian has an SU(4) symmetry for the particular parameter combination $J_{1}=J_{2}=K / 4$. This symmetry reduces to $\mathrm{SU}(2) \times \mathrm{U}(1)$ in an intermediate regime $\left(0<h<h_{c}\right)$, and to $\mathrm{SU}(2)$ at very high magnetic fields where the spins are completely aligned. If the parameter set $\left(J_{1}, J_{2}, K\right)$ is chosen away from the $\mathrm{SU}(4)$ point, the Hamiltonian remains invariant under $\mathrm{SU}(2) \times \mathrm{SU}(2)$. Furthermore, there is a $Z_{2}$ symmetry about $J_{1}=J_{2}$ for vanishing magnetic fields.

Recent studies have concentrated on the phase diagram [1 13$]$ and low-energy excitations [6. [8] of $H$. At the integrable $\mathrm{SU}(4)$ point, the spectrum of excitations has been obtained from the Bethe Ansatz equations [18]. Effective low-energy theories were obtained from bosonization [4.5], and approximate solutions from mean-field theory [19]. In addition, a variety of numerical techniques, including series expansions [1], numerical diagonalization [6], Monte Carlo [7], and density matrix renormalization group [6], has been employed to analyze the ground state and low-energy properties of this Hamiltonian.

In this work, a recently developed density matrix renormalization group technique is applied to study the full spin and orbital dynamics of the one-dimensional spin-orbital model in a magnetic field for arbitrary parameters $\left(J_{1}, J_{2}, K, h\right)$. The advantages of this method are that (i) relatively large Hilbert spaces can be studied (typical system size $\approx 80$ sites), (ii) the full excitation spectra can be obtained, and (iii) there are no constraints concerning low energies, small deviations from the $\mathrm{SU}(4)$ point, or the magnitude of the applied magnetic field. However, the spectra calculated with this method do exhibit some finite-size effects, as 
expected for low-dimensional quantum systems. Here we concentrate on the characteristic features of the dynamical spin and orbital structure factors for the various regimes of $H$. These quantities are defined as

$$
\begin{aligned}
& S(k, \omega)=-\frac{1}{\pi} \lim _{\epsilon \rightarrow 0} \operatorname{Im}\left\langle\Psi_{0}\left|S_{-k}^{z} \frac{1}{\omega+i \epsilon-H+E_{0}} S_{k}^{z}\right| \Psi_{0}\right\rangle, \\
& T(k, \omega)=-\frac{1}{\pi} \lim _{\epsilon \rightarrow 0} \operatorname{Im}\left\langle\Psi_{0}\left|T_{-k}^{z} \frac{1}{\omega+i \epsilon-H+E_{0}} T_{k}^{z}\right| \Psi_{0}\right\rangle,
\end{aligned}
$$

where $\left|\Psi_{0}\right\rangle$ is the ground state, $E_{0}$ its energy, $S_{k}^{z}$ and $T_{k}^{z}$ are the Fourier transforms of the local spin and orbit operators $S_{i}^{z}$ and $T_{i}^{z}$, and a small but finite broadening factor $\epsilon=0.05 J_{1}$ is chosen to represent the poles in $S_{k}^{z}$ and $T_{k}^{z}$.

In general, for the properties of the orbitally degenerate Hamiltonian $H$ to be applicable to candidate inorganic materials such as $\mathrm{Na}_{2} \mathrm{Ti}_{2} \mathrm{Sb}_{2} \mathrm{O}$ and $\mathrm{NaV}_{2} \mathrm{O}_{5}$, the orbital energy levels must be nearly degenerate on the scale of the spin dynamics, which have characteristic

energies in the range of $J_{1} \approx 10-100 \mathrm{meV}$. While external magnetic fields couple directly to the spin degrees of freedom, applied external pressure could be a "dual" perturbation, primarily affecting the orbitals. It would therefore be of great interest to explore the dynamical inelastic neutron scattering response in candidate spin-orbital compounds subject to a magnetic field as well as to external pressure.

In the following section, we discuss briefly the numerical renormalization group method which is used to obtain the spin dynamics of the spin-orbital chain in a magnetic field. In section III, the field-dependent spectra are studied in the different parameter regimes $\left(J_{1}, J_{2}, K, h\right)$. A summary and conclusions are given in section IV.

\section{CALCULATION OF DYNAMICAL PROPERTIES WITH THE DENSITY MATRIX RENORMALIZATION GROUP TECHNIQUE}

The density matrix renormalization group (DMRG) method is known to be a precise numerical technique to calculate the ground state properties of quasi-one-dimensional systems such as chains and ladders. [20] Recently, Hallberg [10] and Kühner and White [11] 
have proposed extensions of this method to extract dynamical correlation functions, based on continued-fraction expansions of the dynamical Green function. In this paper, we use a variant of this technique to calculate the momentum and frequency-dependent spectrum of the spin-orbital model in one spatial dimension.

The zero-temperature, time-dependent correlation function of a momentum-dependent operator $\hat{A}_{k}$ is defined as

$$
A\left(k, t-t^{\prime}\right) \equiv\left\langle\Psi_{0}\left|\hat{A}_{-k}^{\dagger}(t) \hat{A}_{k}\left(t^{\prime}\right)\right| \Psi_{0}\right\rangle
$$

where $\left|\Psi_{0}\right\rangle$ is the ground state. In the following, $\hat{A}_{k}$ corresponds either to the spin operator $S_{k}^{z}$ or to the orbital operator $T_{k}^{z}$. The Fourier transform of this correlation function can be expanded in the form of a continued fraction,

$$
A(k, \omega)=-\frac{1}{\pi} \operatorname{Im} \frac{\left\langle\Psi_{0}\left|\hat{A}_{-k}^{\dagger} \hat{A}_{k}\right| \Psi_{0}\right\rangle}{\omega+i \epsilon-a_{0}-\frac{b_{1}^{2}}{\omega+i \epsilon-a_{1}-\ldots}},
$$

where $\epsilon \rightarrow 0$ is a small broadening factor. The coefficients $a_{n}$ and $b_{n}$ are then obtained from the recursion relation

$$
\left|f_{n+1}\right\rangle=H\left|f_{n}\right\rangle-a_{n}\left|f_{n}\right\rangle-b_{n}^{2}\left|f_{n-1}\right\rangle
$$

with $\left|f_{0}\right\rangle \equiv \hat{A}_{k}\left|\Psi_{0}\right\rangle$ and $b_{0} \equiv 0$. This procedure is very similar to numerical diagonalization calculations. However, there are specific technical problems intrinsic to the DMRG method which need to be addressed. [10,11

One complication arises when open boundary conditions (OBC) are used, which is usual in DMRG calculations to yield the highest possible numerical precision. In this case, the momentum is not a well-defined quantum number, and each momentum-dependent operator $\hat{A}_{k}$ generates wave packets of finite width, centered around $k$. To remedy this problem, Hallberg has used periodic boundary conditions (PBC) to obtain a basis with a good momentum quantum number, whereas Kühner and White have used OBCs and the Parzen filter to eliminate the artificial surface excitations on the surface boundaries. In this paper, we adapt OBCs combined with the Parzen filter to obtain the full dynamical spectra associated with 
$S_{k}^{z}$ and $T_{k}^{z}$. OBCs are known to give more precise ground states than PBCs, and therefore larger clusters can be studied, while the width of the corresponding wave packets is greatly reduced.

Further improvement of the dynamical DMRG method may be obtained by using more target states besides the ground state, implemented with "correction vectors". [11,21] We find that a rather large cut-off dimension of the density matrix is needed to reach convergence in the spin-orbital chain for the target states at higher energy. These convergence problems tend to be exacerbated for excitations away from the dominant wave vectors. However, with the algorithm used in this work, the full structure of the excitation spectrum can be obtained with rather high accuracy, even if only the ground state is kept as a target state, although the relative overlap (matrix elements) with higher-energy states is reduced.

We have tested the algorithm on the spin-1/2 antiferromagnetic Heisenberg chain in a magnetic field. The Hamiltonian for this system corresponds to the parameter choice $\left(J_{1}=J, J_{2}=K=0\right)$ for the spin-orbital model. One observes that the spin-triplet excitation spectrum of this simple model matches very well the exact results from the Bethe Ansatz.

[22] A more detailed discussion of this spectrum will be presented in the next section. Chains with $N=80$ spins were studied. The cut-off factor, i.e. the number of states kept per block, was taken to be $m=160$. We have also used the Lanczos method on lattices of up to 16 sites to check the accuracy of the DMRG results for other non-trivial parameter sets of the spin-orbital chain.

\section{DYNAMICAL SPIN STRUCTURE FACTOR OF THE SPIN-ORBITAL CHAIN}

The rich phase diagram of the one-dimensional spin-orbital model, represented by the Hamiltonian $H$ in Eq. 1, has been the subject of a number of recent studies. [1 3] The main result of these works is summarized in Fig. 1, which we have adapted from Ref. [3]. The various phases can be characterized by spin and orbital quantum numbers $\left(S, S^{z}\right)$ 
and $\left(T, T^{z}\right)$, and by effective Luttinger parameters in each channel, such as gaps $\left(\Delta_{S}, \Delta_{O}\right)$, soliton velocities $\left(v_{S}, v_{O}\right)$, and radii of compactification. For finite values of the spin-orbital interaction $K$, the spin and orbital degrees of freedom are coupled, and the excitation spectra generally contain strongly admixed spin and orbital modes.

Let us first briefly describe the phase diagram of $H$. Phase I is "ferromagnetic" in the spin and orbital channels, i.e. both degrees of freedom are fully polarized, and there is an infinite degeneracy in the ground state. In phase II, the spin degrees of freedom remain polarized, whereas the orbitals are in a quasi-long-range-ordered antiferromagnetic state with a characteristic orbiton velocity $v_{O}$. In phase III, the roles of spins and orbitals are interchanged. Phase IV is fully gapped in all channels $\left(\Delta_{S} \neq 0, \Delta_{O} \neq 0\right)$ because of spontaneous dimerization. In particular, this phase contains a Majumdar-Ghosh point at $J_{1}=J_{2}=3 \mathrm{~K} / 4$ which has a matrix-product ground state. [23,24] It has recently been shown [2.3] that phase $\mathrm{V}$ contains 3 gapless excitations with non-universal, parameter-dependent soliton velocities. At the SU(4)-symmetric point (filled circle in Fig. 1), these three modes have one common velocity, $v=\pi J_{1} / 2=\pi J_{2} / 2=\pi K / 8$. [26]

Let us now turn to the excitation spectra of $H$ in these regimes, starting with phase III where the orbital degrees of freedom are fully polarized. In Fig. 2, the spectrum traced by the dynamical spin structure factor is shown on a lattice of 80 sites, using the parameter set $\left(J_{1}=-J_{2}=K / 2\right)$. The poles have been given a width of $\epsilon=0.05 J_{1}$, and the color scheme highlights the areas of high spectral intensity with bright colors. Since in this phase, the orbital degrees of freedom couple only trivially with the spins, the analysis turns out to be particularly simple. The spin excitation spectrum of this system corresponds to a spin$1 / 2$, antiferromagnetic Heisenberg chain with exchange coupling $J_{1}$, which is dominated by a low-energy 2-spinon continuum (see Fig. 2(a)). [27] The elementary excitations of this system are spin- $1 / 2$ solitons with dispersion $\omega_{\sigma}(q)=3 \pi J_{1} \sin (q) / 4$ for $q \in[0, \pi]$. Thus the 2-spinon continuum has a lower cut-off at $\omega_{l}(k)=3 \pi J_{1}|\sin (k)| / 4$ and an upper cut-off at $\omega_{u}(k)=3 \pi J_{1}|\sin (k / 2)| / 2$. [22, 28] The dominant singularity of the dynamical spin structure 
factor at wave vector $\pi$ diverges according to $S(\pi, \omega) \propto \sqrt{\ln (\omega)} / \omega$ as $\omega \rightarrow 0$.

In a finite magnetic field, this mode becomes incommensurate with nesting vectors $2 k_{F}=$ $\pi(1 \pm 2 m)$, where $m \in[0,1 / 2]$ is the magnetization. This behavior is confirmed by our dynamical DMRG calculation, as shown in Fig. 2(b). Here, a finite magnetization, $m=$ $4 / 40$, leads to an incommensuration with $2 k_{F}=12 \pi / 10$ and $8 \pi / 10$. Because of the relatively large system size $(N=80)$, the spectra appear to be almost continuous, as expected in the thermodynamic limit. However, finite-size effects are still visible, causing gaps and uneven spectral weight distributions at higher energies. Furthermore, the momentum is quantized in 80 slices of size $\pi / 40$. In spite of these limitations, the agreement of the numerical results with the exactly known spectra is encouraging, and gives confidence to proceed to parameter regimes with more complex excitation spectra.

At the $\mathrm{SU}(4)$ point $\left(J_{1}=J_{2}=K / 4\right)$, the spectrum is also known exactly from the Bethe Ansatz. A comparison of the spectra obtained using the DMRG method with the exact results can thus help to further evaluate the accuracy of the numerical approach. In Fig. 3, the spectrum traced by the dynamical spin structure factor is shown on a lattice of 80 sites. From the solution of the Bethe Ansatz equations, it is known that the elementary excitations of the $\mathrm{SU}(4)$ chain are 4 -fold degenerate spin-1/2 spinons $(\sigma)$ and pseudo-spin- $1 / 2$ orbitons $(\tau)$, and 6 -fold degenerate spin-1 or orbital-1 solitons $(\nu)$. 18. The corresponding dispersions of these three elementary excitations are

$$
\begin{aligned}
& \omega_{\sigma}(q)=\frac{J \pi}{2}[\sqrt{2} \cos (-q+3 \pi / 4)+1], q \in[0,3 \pi / 2] \\
& \omega_{\tau}(q)=\frac{J \pi}{2}[\sqrt{2} \cos (-q+\pi / 4)-1], q \in[0, \pi / 2] \\
& \omega_{\nu}(q)=\frac{J \pi}{2} \cos (-q+\pi / 2), q \in[0, \pi]
\end{aligned}
$$

where $J \equiv J_{1}=J_{2}=K / 4$. The spin-triplet excitation spectrum of an $\mathrm{SU}(4)$ chain with $N=4 n$ sites, shown in Fig. 3(a), contains convolutions of elementary $\sigma$ and $\tau$ excitations, as well as $\nu$ excitations. The $z$-component of the dynamical spin structure factor $S^{z}(k, \omega)$ couples directly to the 15 -fold multiplet of $\sigma-\tau$ pairs, the 20 -fold multiplet of $2 \nu$ excitations, and with much smaller matrix elements to the 45 -fold $2 \sigma-\nu$ and the 35 -fold $4 \sigma$ multiplets. 
18

Fig. 3(b) illustrates that the spectrum obtained with the dynamical DMRG traces exactly the two-soliton continua, spanned by $\omega_{\sigma}(q)+\omega_{\tau}\left(q^{\prime}\right)$ and by $\omega_{\nu}(q)+\omega_{\nu}\left(q^{\prime}\right)$. These continua have characteristic low-frequency power-law singularities $\left(S^{z}(k, \omega) \propto \omega^{-\alpha}\right)$ at the Fermi vectors $\pi / 2$ and $3 \pi / 2$, and a weaker divergence at $\pi$. 229 The relatively high spectral intensity seen at larger frequencies and centered around the wave vector $\pi$, can be attributed to a particle-hole excitation in the $\nu$-channel which will be discussed later. We note that the accuracy of the dynamical DMRG method is known to deteriorate at larger energies, and hence the spectral intensities are less reliable in this regime.

When an external magnetic field is applied along the $z$-direction, the spectrum of the SU(4) chain also becomes incommensurate. [6] This effect can be understood quantitatively by considering the splitting of spin-up and spin-down bands due to the applied field. The corresponding nesting vectors depend on the magnetization $m \in[0,1 / 2]$ as $2 k_{F \uparrow}=\pi(1+$ $2 m) / 2,2 k_{F \downarrow}=\pi(1-2 m) / 2$, and $4 k_{F \uparrow}=4 k_{F \downarrow}=\pi(1-2 m)$. This low-frequency behavior is reflected in the finite-size DMRG spectra, shown in Fig. 4, with magnetizations $m=4 / 40$ and $m=12 / 40$. For example, with $m=4 / 40$, the nesting vectors are $6 \pi / 10,4 \pi / 10$, and $8 \pi / 10$, as shown in Figs. 4(a) and (c), whereas for $m=12 / 40$ they are $8 \pi / 10,2 \pi / 10$, and $4 \pi / 10$ respectively (Figs. $4(\mathrm{~b})$ and $(\mathrm{d})$ ).

Furthermore, the overall spectral weight is reduced as the phase space of spin-triplet excitations shrinks with increasing field. At finite magnetic fields, there remains a commensurate soft mode at wave vector $\pi$ in the orbital $\nu$-channel which does not couple to $S(k, \omega)$. It is therefore interesting to examine the orbital excitation spectrum traced out by $T(k, \omega)$. The orbital dynamical structure factor of the $\mathrm{SU}(4)$ chain in a magnetic field is shown in Figs. 4 (c) and (d). In the absence of a magnetic field, the orbital spectrum is identical to the spin spectrum (Fig. 3). In a finite magnetic field there is one orbital mode, the spin-1 $\nu$-channel, which does not become incommensurate, but stays soft at wave vector $\pi$ for all magnetic fields. On the other hand, the strongly mixed $\sigma-\tau$ pairs show incommensuration with the same nesting vectors as for the spin channel. The dominant $\sigma-\tau$ singularities at 
low frequencies occur at $2 k_{F \uparrow}$ for the orbitons and at $2 k_{F \downarrow}$ for the spinons. [6]

The excitation spectra at the Majumdar-Ghosh point $\left(J_{1}=J_{2}=3 \mathrm{~K} / 4\right)$ are shown in Fig. 5. The zero-field ground state at this point in the dimerized phase IV is a doubly degenerate product of spin and orbital singlets with an energy of $-3 J_{1} / 4$ per site and a gap of approximately $3 K / 8$ to the lowest excitations. [1],23,24] The spectrum contains a 2 -soliton continuum of states with minima at wave vectors $k=0$ and $\pi$, and maximum spectral weight at wave vector $\pi$. 24] In addition, there are massive magnon excitations corresponding to Haldane triplet bound states. [25] These are centered at $k=\pi / 2$ and $3 \pi / 2$, indicating a doubling of the real-space unit cell. In Fig. 5(a), we have eliminated the artificial low-energy states which arise from surface excitations at the open boundaries of the 80-site cluster. These states have vanishing spectral weight in the thermodynamic limit.

A finite magnetic field leads to the deconfinement of bound spinons. [30] This is manifested in a lowering and eventual vanishing of the corresponding spin gap when the field exceeds a critical value, which for the Majumdar-Ghosh point is at $h_{c 1} \approx 3 K / 8$. In Fig. $5(\mathrm{~b})$, the spin excitation spectrum is shown in this incommensurate regime $\left(h_{c 1}<h<h_{c 2}\right)$ at a magnetization $m=4 / 40$. The dominant low-energy singularities of the spin-1 magnons occur at wave vectors $4 k_{F}=\pi(1 \pm 2 m)$. The 2-spinon continuum at higher energies appears to be almost unaffected by the finite magnetic field. Interestingly, a strong low-energy singularity at $k=\pi$ in the orbital channel is induced by the applied field, as observed in Fig. 5(c), which is indicative of an effective attractive potential between the incommensurate spin-1 and the commensurate orbital-1 $\nu$-type solitons in this case. This magnetic-field-induced two-component Luttinger Liquid behavior, with the orbital channel remaining commensurate and the spin channel becoming incommensurate, has also been observed in recent bosonization studies of the spin-orbital chain. [8,9]

Finally, let us turn to the gapless phase V. In Figs. 6 and 7, the spin and orbital excitation spectra are shown at $J_{1}=J_{2}=K / 8$ and at $J_{1}=J_{2}=0$. As $K \rightarrow 0$, a prominent high-energy excitation emerges, centered at wave vector $\pi$ and frequency $\omega \approx 0.8 K$. This excitation carries the quantum numbers $(S, T)=(0,1)$ and $(1,0)$ which indicates a particle- 
hole-type bound state, involving a spin singlet and an orbital triplet or vice versa. From finite-size scaling, we observe that the energy gap between the ground state and this level increases with system size. If $J_{1}=J_{2}=0$, the eigenstates of the $K$ term can be found exactly on a 4 -site cluster. The ground state with energy $-3 K / 4$ and the excitons with energy 0 are in this case

$$
\begin{aligned}
|G S\rangle & =\sum_{i=1}^{4}(-1)^{i} \mid\left(t_{i, O}^{+} t_{i+2, O}^{-}\right)\left(s_{i, S} s_{i+2, S}\right)-\left(s_{i, O} s_{i+2, O}\right)\left(t_{i, S}^{+} t_{i+2, S}^{-}\right) \\
& \left.+\left(t_{i+1, O}^{-} t_{i+3, O}^{+}\right)\left(t_{i, S}^{+} t_{i+2, S}^{-}\right)-\left(t_{i, O}^{+} t_{i+2, O}^{-}\right)\left(t_{i+1, S}^{-} t_{i+3, S}^{+}\right)\right\rangle \\
|\pi, S\rangle & =\sum_{i=1}^{4}\left|\left(t_{i, O}^{+} t_{i+2, O}^{-}\right)\left(t_{i, S}^{+} s_{i+2, S}\right)\right\rangle \text { and }|\pi, O\rangle=\sum_{i=1}^{4}\left|\left(t_{i, S}^{+} t_{i+2, S}^{-}\right)\left(t_{i, O}^{+} s_{i+2, O}\right)\right\rangle
\end{aligned}
$$

where, for example, $t_{i, S}^{+}$denotes an up-up triplet state in the spin channel, involving spins at sites $i$ and $i+1$, and $s_{i+2, O}$ denotes a singlet in the orbital channel at sites $i+2$ and $i+3$. This high-energy bound state is observed in the entire phase $\mathrm{V}$, but is most prominent in the strong- $K$ limit.

The magnetic-field dependence of the spectra in phase $\mathrm{V}$ can be analyzed analogously to the SU(4) limit. In particular, the zero-energy modes can be understood within the band picture discussed above, with nesting instabilities at $2 k_{F \uparrow}, 2 k_{F \downarrow}$, and $4 k_{F \uparrow}=4 k_{F \downarrow}$. The finitesize gaps in these spectra appear to be most obvious at the critical point, and become less severe as $J_{1}, J_{2} \rightarrow 0$.

\section{SUMMARY}

In summary, the spin and orbital excitation spectra of the spin-orbital chain were studied in various regimes of the model Hamiltonian $H$, given in Eq. 1. The characteristic dynamical response depends strongly on the parameter set $\left(J_{1}, J_{2}, K\right)$ and on the applied field $h$. In phases II and III with either fully polarized spin or orbital degrees of freedom, the remaining

gapless mode has the characteristic spectral response of a (pseudo)-spin-1/2 Heisenberg chain. On the other hand, in the dimerized regime (phase IV), a finite magnetic field is needed to close the spin gap. Interestingly, an additional orbital mode is found to become 
gapless at the commensurate wave vector $\pi$ in the partially spin-polarized regime $\left(h_{c 1}<\right.$ $\left.h<h_{c 2}\right)$, whereas the Fermi momenta of the incommensurate gapless spin modes depend on the magnetization via $2 k_{F}=\pi(1-2 m)$. In the gapless regime (phase $\mathrm{V}$ ), there are three elementary gapless excitations. The dynamical response consists of various overlapping twoparticle continua, arising from pairs of these elementary excitations. At higher energies, a particle-hole bound state whose origin lies in the biquadratic $K$-term, is found to dominate the spectrum.

We are greatful to Andreas Honecker, Till Kühner, Bruce Normand, and Edmond Orignac for useful discussions, and we thank Till Kühner for valuable help in developing the dynamical DMRG algorithm. S. H. acknowledges the Zumberge foundation for financial support. 


\section{REFERENCES}

[1] S. K. Pati, R. R. P. Singh, and D. I. Khomskii, Phys. Rev. Lett. 81, 5406 (1998).

[2] Y. Yamashita, N. Shibata, K. Ueda, J. Phys. Soc. Jpn. 61, 242 (2000).

[3] C. Itoi, S. Qin, and I. Affleck, Phys. Rev. B 61, 6747 (2000).

[4] P. Azaria, A. O. Gogolin, P. Lecheminant, and A. A. Nersesyan, Phys. Rev. Lett. 83, $624(1999)$.

[5] P. Azaria, E. Boulat, and P. Lecheminant, Phys. Rev. B 61, 12112 (2000).

[6] Y. Yamashita, N. Shibata, K. Ueda, Phys. Rev. B 61, 4012 (2000); Y. Yamashita, N. Shibata, K. Ueda, Phys. Rev. B 58, 9114 (1998).

[7] B. Frischmuth, F. Mila, and M. Troyer, Phys. Rev. Lett. 82, 835 (1999).

[8] E. Orignac, R. Citro, and N. Andrei, Phys. Rev. B 61, 11533 (2000).

[9] Y.-L. Lee and Y.-W. Lee, Phys. Rev. B 61, 6765 (2000).

[10] K. A. Hallberg, Phys. Rev. B 52, R9872 (1995).

[11] T. D. Kühner and S. R. White, Phys. Rev. B 60, 3351 (1999).

[12] E. Axtell, T. Ozawa, S. Kauzlarich, and R. R. P. Singh, J. Solid State Chem. 134, 423 (1997).

[13] M. Isobe and Y. Ueda, J. Phys. Soc. Jpn. 65, 1178 (1996).

[14] Y. Fujii, H. Nakao, T. Yosihama, M. Nishi, K. Nakajima, K. Kakurai, M. Isobe, Y. Ueda, and H. Sawa, J. Phys. Soc. Jpn. 66, 326 (1997).

[15] H. Smolinski, C. Gros, W. Weber, U. Peuchart, G. Roth, M. Weiden, and C. Geibel, Phys. Rev. Lett. 80, 5164 (1998).

[16] D. Augier, D. Poilblanc, S. Haas, A. Delia, and E. Dagotto, Phys. Rev. B 56, R5732 
(1997).

[17] K. I. Kugel and D. I. Khomskii, Usp. Fiz Nauk 136, 621 (1982), [Sov. Phys. Ups. 25, $231(1982)]$.

[18] Y. Q. Li, M. Ma, D. N. Shi, and F. C. Zhang, Phys. Rev. Lett. 81, 3527 (1998): Y. Q. Li, M. Ma, D. N. Shi, and F. C. Zhang, Phys. Rev. B 60, 12781 (1999).

[19] J. van der Brink, W. Stekelenburg, D.I. Khomskii, G.A. Sawatzky, and K.I. Kugel, Phys. Rev. B 58, 10276 (1998).

[20] S.R. White, Phys. Rev. Lett. 69, 2863 (1992); S. R. White, Phys. Rev. B 48, 10345 (1993); I. Peschel, X. Wang, M. Kaulke, and K. Hallberg (eds.), Density-Matrix Renormalization, Lecture Notes in Physics 528 (Springer, Berlin 1999).

[21] S. K. Pati, S. Ramasesha, Z. Shuai, and J. L. Bredas, Phys. Rev. B. 59, 14827 (1999).

[22] J. des Cloiseaux and J.J. Pearson, Phys. Rev. 128, 2131 (1962).

[23] C. K. Majumdar and D. K. Ghosh, J. Math. Phys. (N.Y.) 10, 1399 (1969).

[24] A.K. Kolezhuk and H.J. Mikeska, Phys. Rev. Lett. 80, 2709 (1998).

[25] F. D. M. Haldane, Phys. Lett. 93A, 464 (1983); F. D. M. Haldane, Phys. Rev. Lett. 50, 1153 (1983).

[26] B. Sutherland, Phys. Rev. B 12, 3795 (1975).

[27] A. H. Bougourzi, M. Karbach, and G. Müller, Phys. Rev. B 57, 11429 (1998).

[28] S. Haas, J. Riera, and E. Dagotto, Phys. Rev. B 48, 13174 (1993).

[29] Logarithmic corrections to these low-energy singularities are expected to occur. However, a comprehensive analysis of the scaling behavior, including the effects of marginally relevant currents, is beyond the scope of this work, and will be addressed in a future publication. 
[30] W. Yu and S. Haas, to be published in Phys. Rev. B, cond-mat/9909093. 


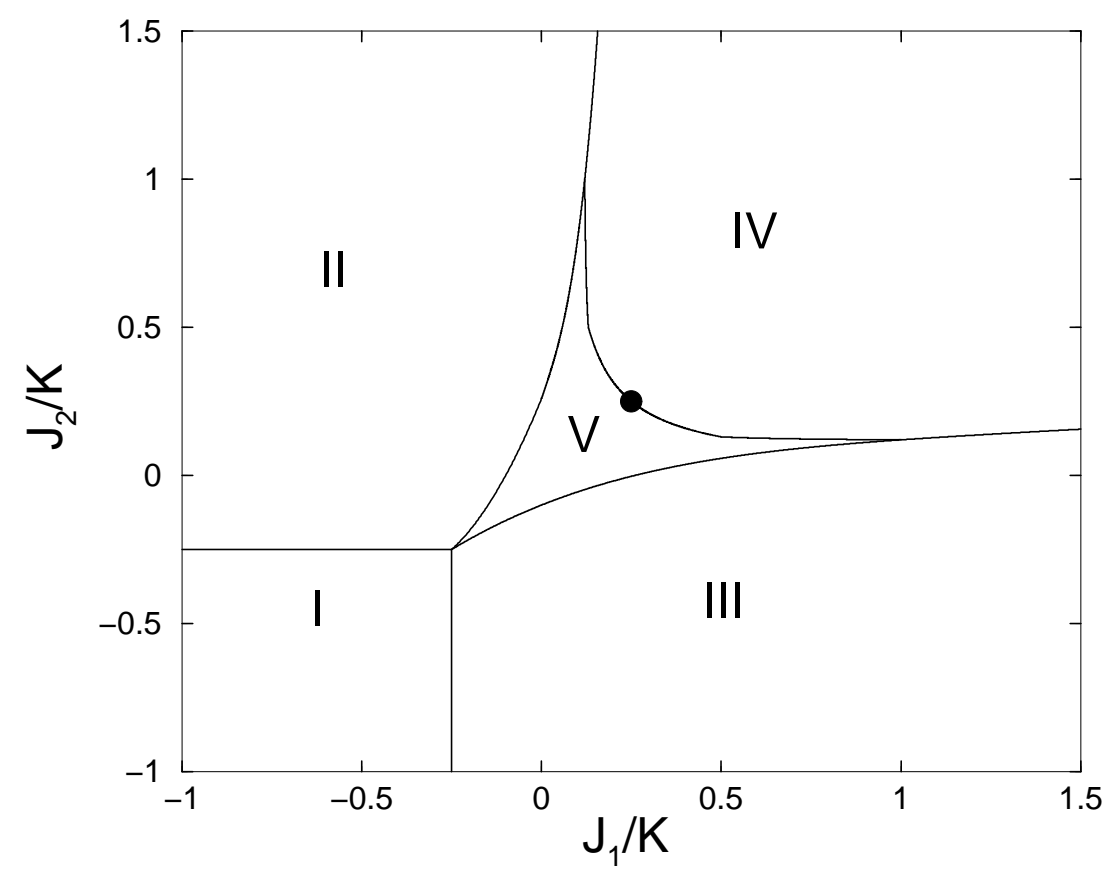

FIG. 1. Phase diagram of the one-dimensional spin-orbital model, adapted from Ref. 3. Phase I: spins and orbitals are fully polarized. Phases II and III: one of the channels is fully polarized, whereas the other is a quasi-long-range-ordered antiferromagnet. Phase IV: dimerized regime. Phase V: gapless regime. Circle: SU(4)-symmetric point. 

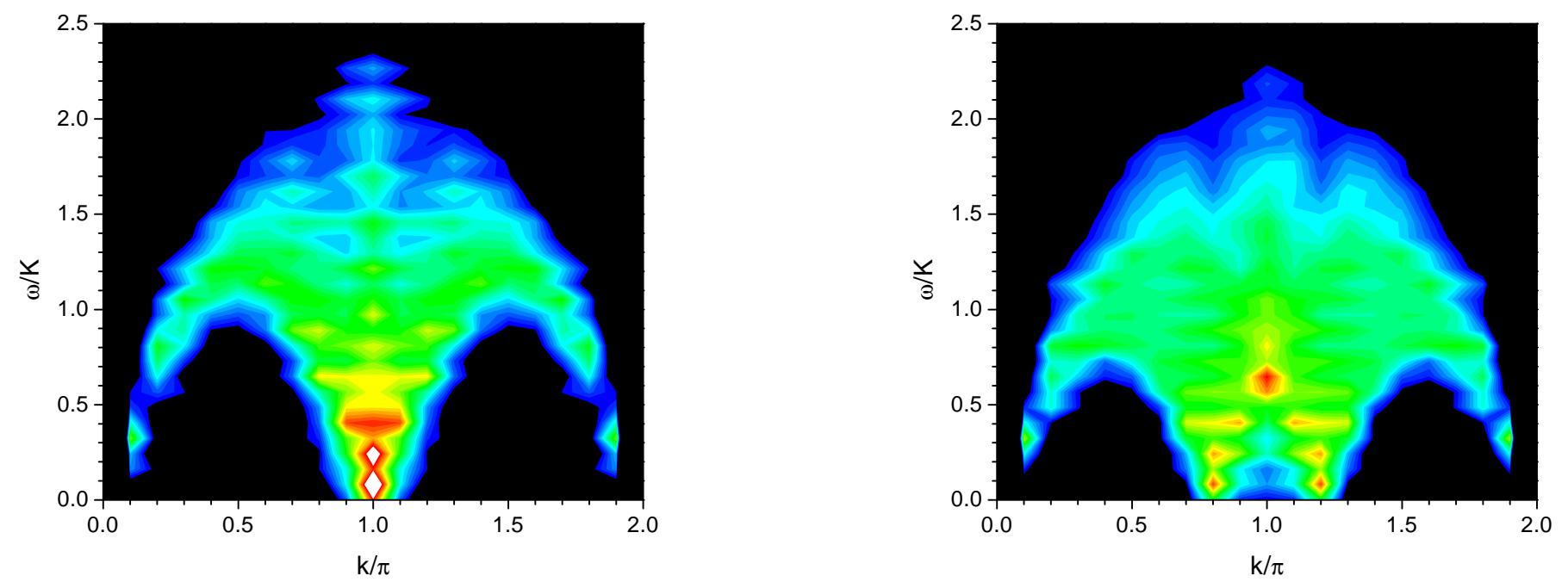

FIG. 2. Spin excitation spectrum of the spin-orbital chain at $\left(J_{1}=-J_{2}=K / 2\right)$, obtained with dynamical DMRG on a 80-site cluster. (a) zero magnetic field $m=0$, and (b) $m=4 / 40$.
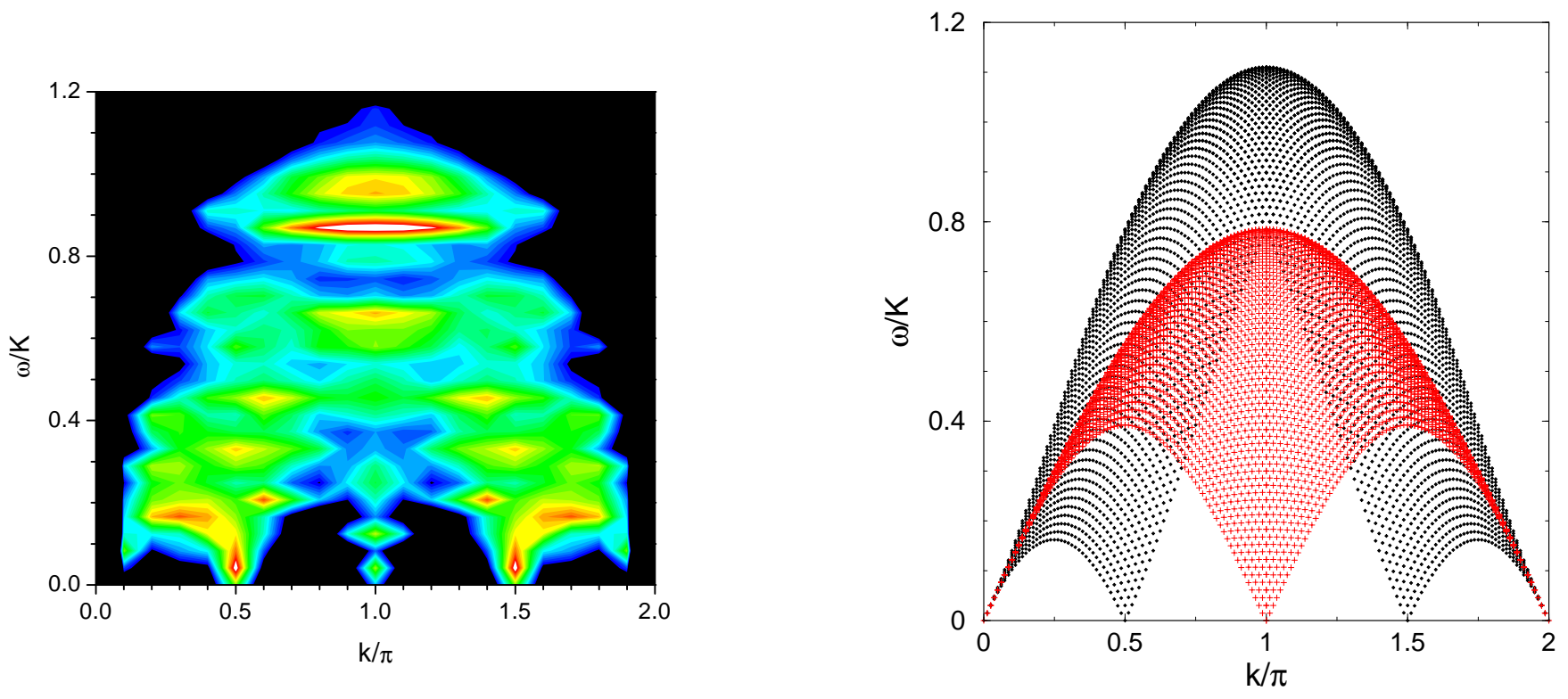

FIG. 3. (a) Dynamical spin structure factor of the SU(4)-symmetric spin-orbital chain. This spectrum was calculated using the dynamical DMRG technique on an 80-site cluster. (b) Spin-triplet spectra composed of $\sigma-\tau$ pairs (dots) and $2 \nu$ pairs (pluses), obtained from convolutions of the single-soliton dispersions, which are known exactly from the Bethe Ansatz solution. 

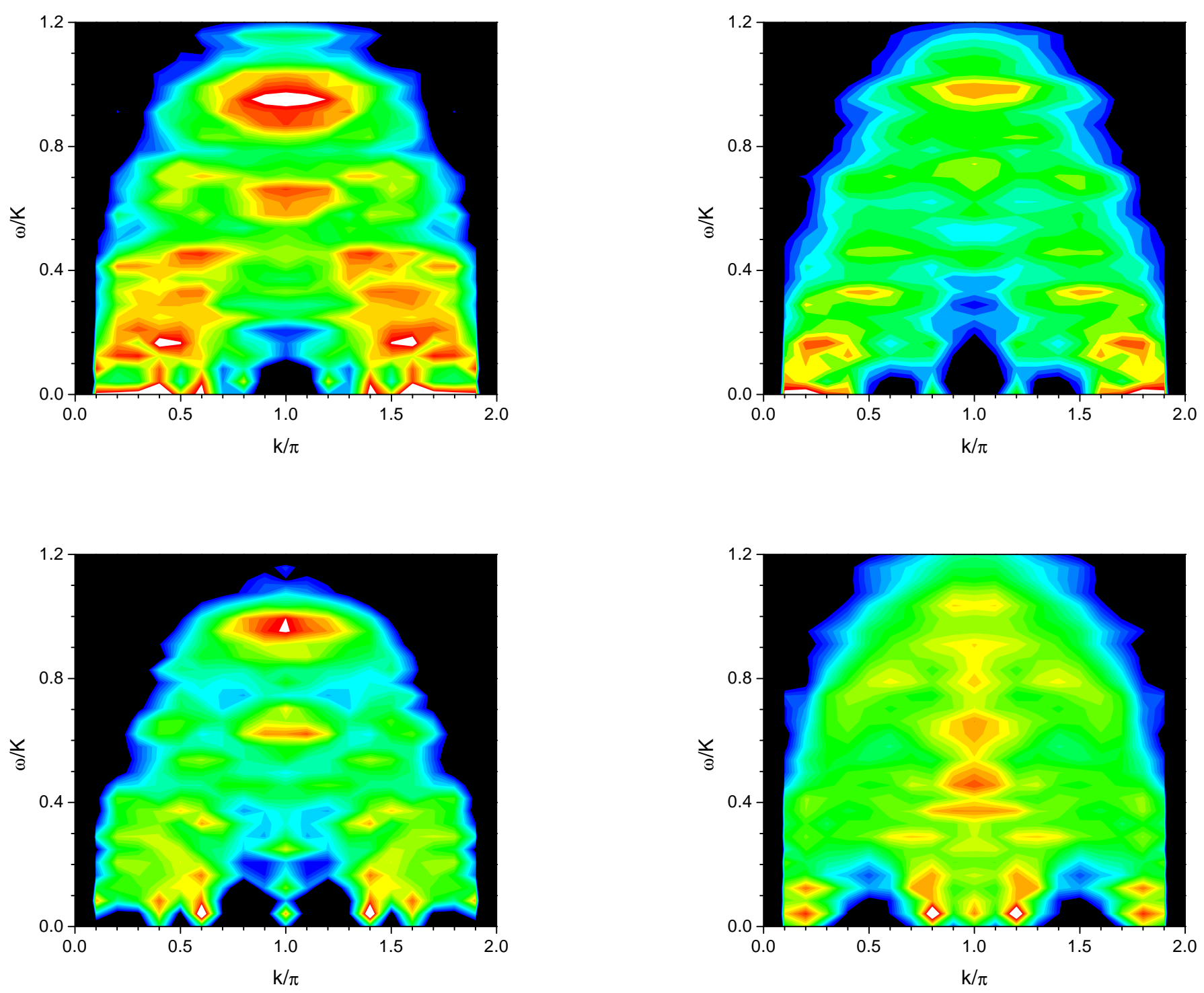

FIG. 4. Spin and orbital excitation spectra of the SU(4) chain in a magnetic field. (a) (upper left) spin dynamics at $m=4 / 40$, (b) (upper right) spin dynamics at $m=12 / 40$, (c) (lower left) orbital dynamics at $m=4 / 40$, and (d) (lower right) orbital dynamics at $m=12 / 40$. 

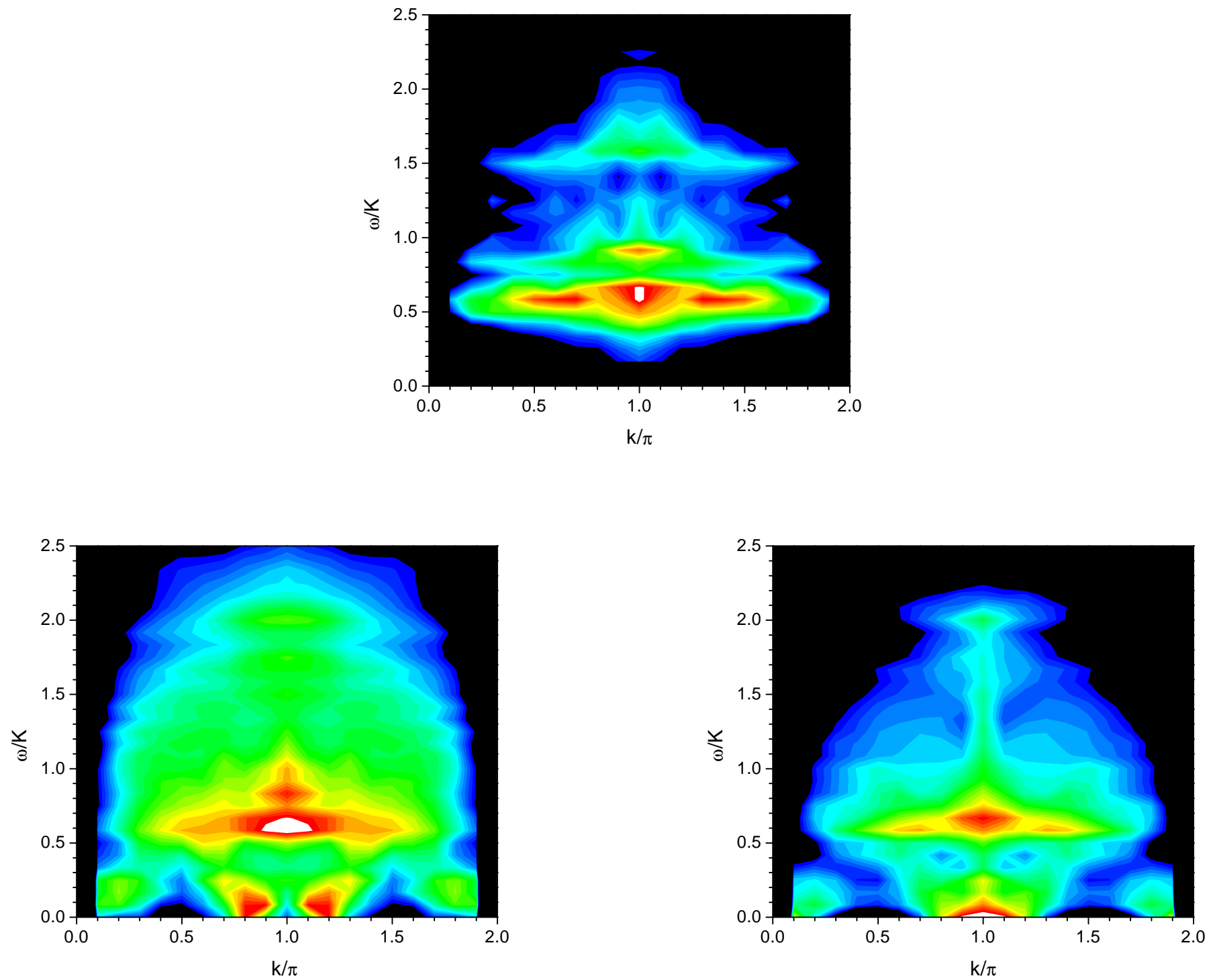

FIG. 5. Spin and orbital excitation spectra of the spin-orbital chain at the Majumdar-Ghosh point $\left(J_{1}=J_{2}=3 K / 4\right)$. (a) (upper) spin and orbital dynamics at $m=0$, (b) (lower left) spin dynamics at $m=4 / 40$, and (c) (lower right) orbital dynamics at $m=4 / 40$. 

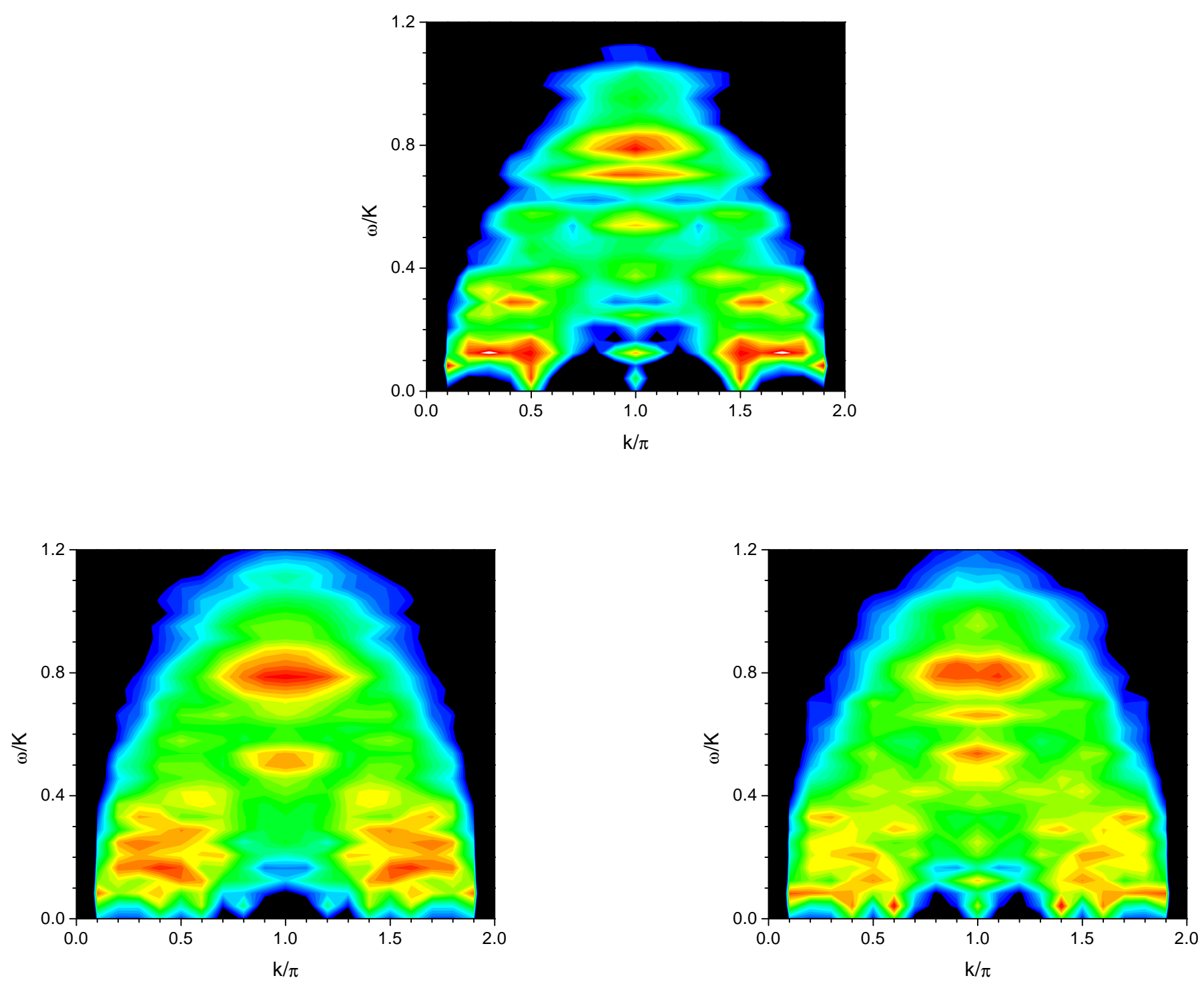

FIG. 6. Spin and orbital excitation spectra of the spin-orbital chain at $\left(J_{1}=J_{2}=K / 8\right)$. (a) (upper) spin and orbital dynamics at $m=0$, (b) (lower left) spin dynamics at $m=4 / 40$, and (c) (lower right) orbital dynamics at $m=4 / 40$. 

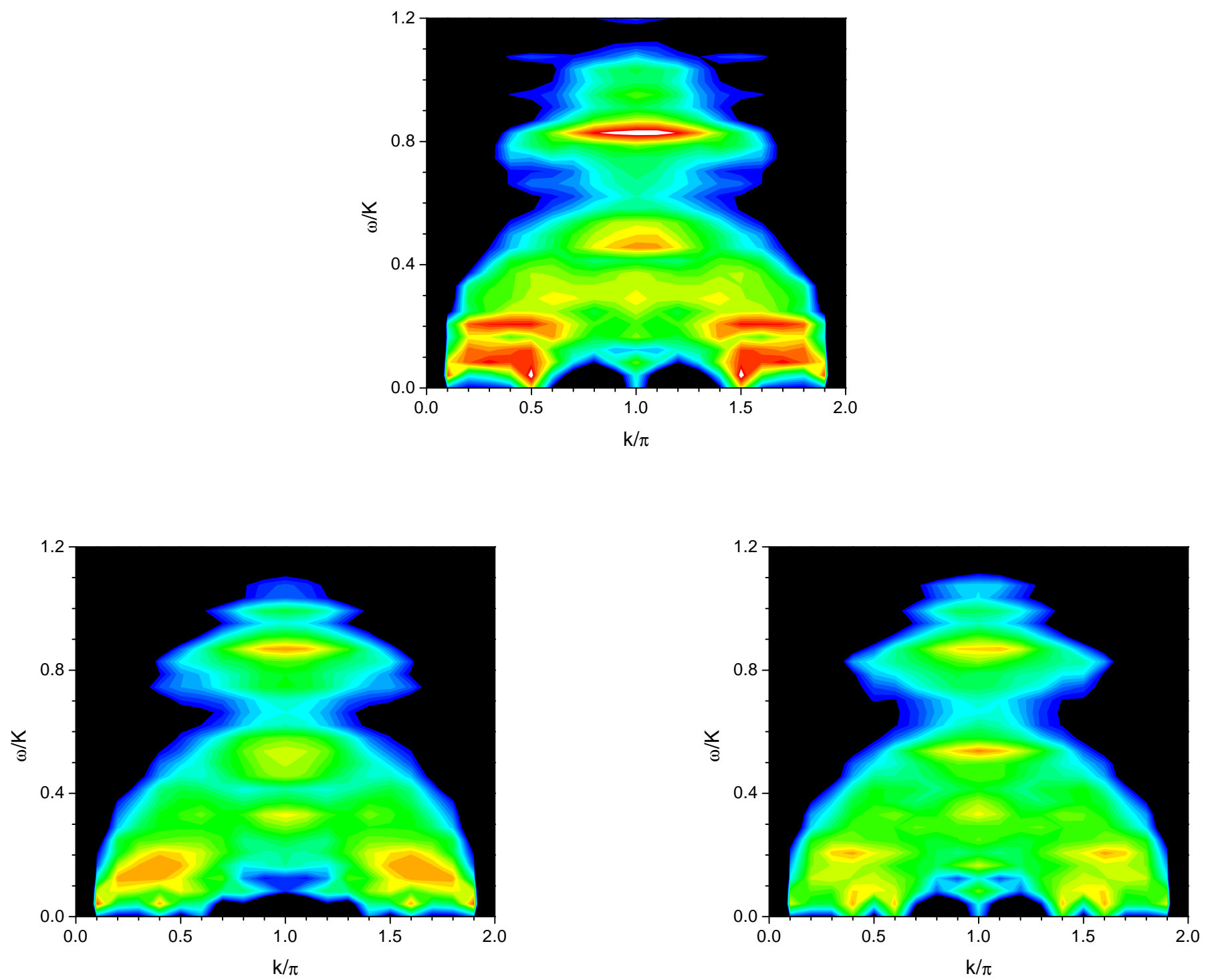

FIG. 7. Spin and orbital excitation spectra of the spin-orbital chain at $\left(J_{1}=J_{2}=0\right)$. (a) (upper) spin and orbital dynamics at $m=0$, (b) (lower left) spin dynamics at $m=4 / 40$, and (c) (lower right) orbital dynamics at $m=4 / 40$. 